\section{(C) OPEN ACCESS}

Handling editor Josef $S$ Smolen

- Additional material is published online only. To view please visit the journal online (http://dx.doi.org/10.1136/ annrheumdis-2019-215086).

${ }^{1}$ Academic Rheumatology, University of Nottingham, Nottingham, UK

${ }^{2}$ Division of Epidemiology and Public Health, University of Nottingham, Nottingham, UK ${ }^{3}$ Primary Care Centre Versus Arthritis, Keele University, Keele, UK

${ }^{4}$ Nottingham NIHR Biomedical Research Centre, Nottingham, UK

Correspondence to Dr Georgina Nakafero, Academic Rheumatology, University of Nottingham, Nottingham NG5 1PB, UK; georgina.nakafero@ nottingham.ac.uk

Received 17 January 2019 Revised 25 March 2019 Accepted 7 April 2019 Published Online First 29 April 2019

\title{
Association between inactivated influenza vaccine and primary care consultations for autoimmune rheumatic disease flares: a self-controlled case series study using data from the Clinical Practice Research Datalink
}

\author{
Georgina Nakafero, 1 Matthew J Grainge, ${ }^{2}$ Puja R Myles, ${ }^{2}$ Christian D Mallen, ${ }^{3}$ \\ Weiya Zhang, ${ }^{1}$ Michael Doherty, ${ }^{1}$ Jonathan S Nguyen-Van-Tam, ${ }^{2}$ Abhishek Abhishek ${ }^{1,4}$
}

Objectives To examine the association between inactivated influenza vaccine (IIV) administration and primary care consultation for joint pain, rheumatoid arthritis (RA) flare, corticosteroid prescription, vasculitis and unexplained fever in people with autoimmune rheumatic diseases (AIRDs).

Methods We undertook within-person comparisons using self-controlled case-series methodology. AIRD cases who received the IIV and had an outcome of interest in the same influenza cycle were ascertained in Clinical Practice Research Datalink. The influenza cycle was partitioned into exposure periods (1-14 days prevaccination and $0-14,15-30,31-60$ and $61-90$ days postvaccination), with the remaining time-period classified as non-exposed. Incidence rate ratios (IRR) and 95\% Cl for different outcomes were calculated.

Results Data for 14928 AIRD cases (69\% women, $80 \%$ with RA) were included. There was no evidence for association between vaccination and primary care consultation for RA flare, corticosteroid prescription, fever or vasculitis. On the contrary, vaccination associated with reduced primary care consultation for joint pain in the subsequent 90 days (IRR 0.91 (95\% Cl 0.87 to 0.94)).

Conclusion This study found no evidence for a significant association between vaccination and primary care consultation for most surrogates of increased disease activity or vaccine adverse-effects in people with AIRDs. It adds to the accumulating evidence to support influenza vaccination in AIRDs.

\section{INTRODUCTION}

Autoimmune rheumatic diseases (AIRDs) such as rheumatoid arthritis (RA) associate with increased risk of influenza and its complications. ${ }^{1}$ Even though inactivated influenza vaccine (IIV) has clinical and serological effectiveness in AIRDs, its uptake remains suboptimal. ${ }^{2-4}$ For instance, when AIRDs were the sole indication for vaccination, $49 \%$ and $59 \%$ people aged $18-44$ and $45-64$ years, respectively, were vaccinated in the 2015-2016 influenza season in the $\mathrm{UK},{ }^{5}$ with lower vaccine uptake reported in Germany. ${ }^{6}$

Barriers to IIV include scepticism about effectiveness, concerns about side effects or disease flare and reports of vaccination triggering AIRDs such as vasculitis. $^{7-11}$ Trials assessing serological response to

\section{Key messages}

What is already known about this subject?

- Inactivated influenza vaccine is recommended in patients with autoimmune rheumatic diseases to minimise the increased risk of influenza and its complications in this population.

- Concerns about influenza vaccine associating with increased risk of autoimmune rheumatic disease (AIRD) activity and anecdotal reports of the influenza vaccine triggering diseases such as vasculitis are barriers to seasonal influenza vaccination.

What does this study add?

- Seasonal influenza vaccination is not associated with AIRD flare and vasculitis.

How might this impact on clinical practice or future developments?

- This study provides new data on the safety of influenza vaccine in people with AIRDs and adds to the accumulating evidence to support seasonal influenza vaccination in this population.

IIV report stable disease activity following vaccination provided disease-modifying antirheumatic drugs (DMARDs) treatment is continued. ${ }^{12}{ }^{13}$ However, these studies typically include people with stable disease, and, to the best of our knowledge, a realworld study evaluating the effect of IIV on AIRDs has not been performed. Thus, the objectives of this study were to investigate the association between IIV administration and primary care consultation for joint pain, RA flare, new oral corticosteroid prescription and potential vaccine adverse effects, such as vasculitis and non-infective fever.

\section{METHODS}

\section{Data source}

Data were extracted from the Clinical Practice Research Datalink (CPRD). ${ }^{14}$ CPRD is a longitudinal database of anonymised health records of $>15$ million people registered in $>700$ general 
practices in the UK. Participants are representative of the UK population. ${ }^{14}$ It contains details of demographics, diagnoses, immunisations, prescriptions and lifestyle factors.

\section{Study design}

Self-controlled case series (SCCS) was developed for assessing associations between exposures and outcomes using data from participants who develop an outcome of interest and is an accepted methodology for vaccine safety studies. ${ }^{15} 16$ It has the advantage of being unaffected by between-person confounding as each participant acts as their own control. However, it does not account for time varying covariates such as season which vary between the unexposed and exposed periods.

\section{Source population}

Adults aged $\geq 18$ years with RA, spondyloarthritis $(\mathrm{SpA})$ or systemic lupus erythematosus (SLE) and prescribed DMARDs. ${ }^{3}$

\section{Study period}

The study period was 1 September 2006 to 31 of August 2016. This was partitioned into 10 influenza cycles, beginning on 1 September of 1 year and ending on 31 August the subsequent year. Due to the use of non-standard monovalent vaccine alongside trivalent IIV during 2009-2010 pandemic year, data for this year were excluded. Observation periods for each year were censored if death, emigration from general practice or last collection of data from general practice occurred before 31 August of the subsequent year.

\section{Exposure and outcomes}

Vaccination, the exposure of interest, was defined using Read $\operatorname{codes}^{5}$ and event dates. Influenza cycles in which a patient was coded as having received the IIV elsewhere, for example, at work for health care professionals or in community pharmacies were excluded from the analysis as the date of vaccination outside primary care is not recorded in the CPRD. For cases with $>1$ entry for vaccination in an influenza cycle, the earliest record was retained.

The outcomes were primary care consultation for:

A. Surrogates of disease activity: joint pain, flare of RA and new corticosteroid prescriptions.

B. Vaccine hypersensitivity: vasculitis, non-infective fever. Only the first Read code for vasculitis was considered since it is a chronic illness, and, participants diagnosed with vasculitis before study entry were excluded.

See online supplementary material for details.

\section{Exposure and unexposed periods}

The influenza cycle was divided into unexposed and exposed periods, and the latter was further categorised into smaller time periods (figure 1). The first cut-off was selected at 14-day postvaccination as it takes 2 weeks for the IIV to induce a serological response, and, we hypothesised that this period of immune reconstitution was most likely to associate with disease activity. ${ }^{17}$ The 14-day period immediately preceding vaccination was excluded from the baseline period to minimise confounding due to healthy vaccinee effect. ${ }^{18}$

\section{Statistical analyses}

SCCS involves fitting a Poisson model conditioned on the number of events and calculates incidence rate ratios and 95\% CI for each exposure period. Distinct SCCS analysis data sets were drawn for each outcome. Each participant contributed data from one randomly selected eligible influenza cycle in which both vaccination and an outcome of interest occurred (online supplementary table S1). We analysed data from single randomly selected influenza cycle since people who develop an adverse outcome temporally closely related to vaccination, for example, joint pain within 1-2 weeks of vaccination, will be less likely to attend for repeat vaccinations compared to those who have such an event after a longer time period. Thus, if vaccination was to cause an adverse effect, including data from all influenza cycles in which an outcome occurred for a study participant would introduce differential bias. Except for vasculitis, $>1$ event of the same type in an influenza cycle were considered as recurrent episodes provided the interval between any two consultations was $\geq 15$ days.

As corticosteroids are prescribed for many reasons, we performed sensitivity analysis restricted to corticosteroid prescription on the same day on which there is a primary care consultation for joint pain or RA flare. All analyses were carried out using Stata V.14.

\section{RESULTS}

Data for 14928 AIRD cases with $\geq 1$ outcome of interest in an influenza cycle in which they received the IIV were included. Of these, 11953 (80.07\%) had RA, 2347 (15.72\%) had SpA and $628(4.21 \%)$ had SLE. The majority were female $(68.5 \%)$ and

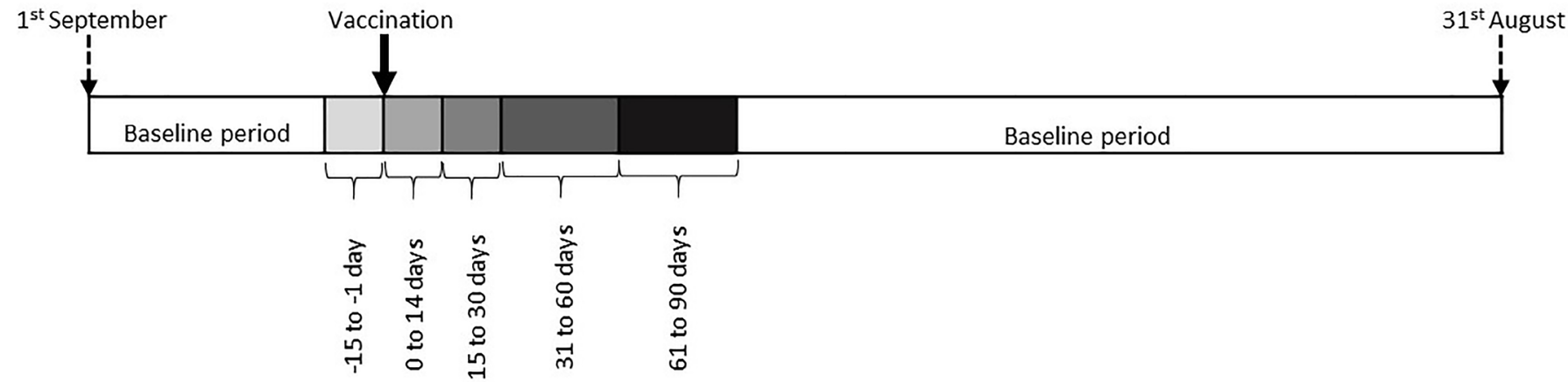

Figure 1 Influenza cycle divided into baseline, prevaccination and postvaccination periods. The baseline extended from latest of diagnosis date or 1st September in index year to 15 days pre-vaccination, and from 90 days post-vaccination to earliest of 31st August of the next year, date of leaving GP surgery, date of death, or latest date of data collection. Exposed period extended from vaccination to 90 days later,and was categorised as illustrated above. 
Miscellaneous

\begin{tabular}{|c|c|c|c|c|c|}
\hline Outcome & Risk period & Events (n) & Person-time (days) & $\operatorname{IRR}(95 \% \mathrm{CI})^{*}$ & P value \\
\hline \multirow[t]{7}{*}{ Joint pain } & Unexposed & 9977 & 2712373 & 1.00 & $<0.001$ \\
\hline & 15 days prevaccination & 788 & 160775 & 1.29 (1.20 to 1.39$)$ & \\
\hline & Postvaccination intervals & & & & \\
\hline & $0-14$ days & 479 & 150314 & 0.84 (0.77 to 0.92$)$ & $<0.001$ \\
\hline & $15-30$ days & 567 & 160842 & 0.94 (0.86 to 1.02$)$ & 0.127 \\
\hline & $31-60$ days & 1121 & 321024 & 0.93 (0.88 to 0.99$)$ & 0.025 \\
\hline & $61-90$ days & 1069 & 319890 & $0.90(0.84$ to 0.96$)$ & 0.001 \\
\hline \multirow{7}{*}{$\begin{array}{l}\text { Corticosteroid } \\
\text { prescription }\end{array}$} & Unexposed & 9470 & 2266070 & 1.00 & $<0.001$ \\
\hline & 15 days prevaccination & 704 & 135493 & 1.21 (1.12 to 1.31$)$ & \\
\hline & Postvaccination intervals & & & & \\
\hline & $0-14$ days & 539 & 126625 & 1.00 (0.91 to 1.09 ) & 0.924 \\
\hline & $15-30$ days & 554 & 135515 & 0.96 (0.88 to 1.04$)$ & 0.338 \\
\hline & $31-60$ days & 1151 & 270109 & 1.00 (0.95 to 1.07$)$ & 0.876 \\
\hline & $61-90$ days & 1182 & 268968 & 1.04 (0.98 to 1.11 ) & 0.184 \\
\hline \multirow[t]{7}{*}{ RA flare } & Unexposed & 460 & 153247 & 1.00 & 0.785 \\
\hline & 15 days prevaccination & 24 & 8957 & 0.95 (0.64 to 1.41$)$ & \\
\hline & Postvaccination intervals & & & & \\
\hline & $0-14$ days & 27 & 8400 & 0.97 (0.65 to 1.45$)$ & 0.888 \\
\hline & $15-30$ days & 29 & 9000 & 1.12 (0.78 to 1.62 & 0.525 \\
\hline & $31-60$ days & 59 & 18000 & 1.06 (0.80 to 1.39$)$ & 0.693 \\
\hline & $61-90$ days & 46 & 17978 & 0.81 (0.59 to 1.10$)$ & 0.180 \\
\hline
\end{tabular}

*Statistically significant results are in bold $(p<0.05)$.

IIV, inactivated influenza vaccine; IRR, incidence rate ratio; RA, rheumatoid arthritis.

their mean (SD) age was 59 (14) years. Vaccination associated with fewer primary care consultations for joint pain with the magnitude of reduction broadly consistent across the four risk periods (table 1). When data for the first 30 days were pooled together, vaccination associated with fewer primary care consultations for joint pain (IRR 0.89 (95\% CI 0.84 to 0.9)). The 14-day prevaccination period associated with significantly more primary care consultations for joint pain and new corticosteroid prescriptions (table 1). The median (IQR) corticosteroid dose was $10(5-30) \mathrm{mg} /$ day prednisolone equivalents, data available for $54 \%$ prescriptions.
There were no significant associations between vaccination and other adverse outcomes in this study (tables 1 and 2). On sensitivity analysis, vaccination was not associated with new corticosteroid prescription on the same day on which there was a primary care consultation for either RA flare or joint pain (online supplementary table S2).

\section{DISCUSSION}

This study reports no significant associations between IIV administration and new primary-care corticosteroid prescriptions or primary care consultations for vasculitis and

Table 2 Association between inactivated influenza vaccine, vasculitis and unexplained fever*

\begin{tabular}{|c|c|c|c|c|c|}
\hline Outcome & Risk period & Events (n) & Person-time (days) & Incidence rate ratio $(95 \% \mathrm{Cl})$ & $P$ values \\
\hline \multirow[t]{7}{*}{ Vasculitis } & Unexposed & 89 & 30936 & 1.00 & 0.906 \\
\hline & 15 days prevaccination & 5 & 1815 & 0.95 (0.38 to 2.33 ) & \\
\hline & Postvaccination intervals & & & & \\
\hline & $0-14$ days & $X^{1}$ & 1694 & 0.41 (0.10 to 1.65$)$ & 0.207 \\
\hline & $15-30$ days & 5 & 1815 & 0.95 (0.38 to 2.33$)$ & 0.906 \\
\hline & $31-60$ days & 8 & 3630 & 0.76 (0.37 to 1.56$)$ & 0.453 \\
\hline & $61-90$ days & 12 & 3630 & 1.14 (0.62 to 2.08$)$ & 0.677 \\
\hline Unexplained & Unexposed & 92 & 30735 & 1.00 & 0.507 \\
\hline \multirow[t]{6}{*}{ Fever } & 15 days prevaccination & $X^{1}$ & 1819 & 0.71 (0.26 to 1.94$)$ & \\
\hline & Postvaccination intervals & & & & \\
\hline & $0-14$ days & $x^{1}$ & 1708 & 0.71 (0.26 to 1.94$)$ & 0.589 \\
\hline & $15-30$ days & 6 & 1830 & 1.06 (0.28 to 2.07$)$ & 0.887 \\
\hline & $31-60$ days & 5 & 3650 & $0.45(0.18$ to 1.10$)$ & 0.079 \\
\hline & $61-90$ days & 12 & 3630 & 1.09 (0.60 to 1.99$)$ & 0.780 \\
\hline
\end{tabular}

$\mathrm{X}^{1}$ fewer than five events in each cell, data suppressed according to Clinical Practice Research Datalink policy.

* Unexplained fever was defined as fever not due to a known cause, for example, infection. 
non-infective fever. However, there was a negative association between vaccination and primary care consultations for joint pain upto 90 days postvaccination. Further research is required to understand the underlying mechanism. It is unlikely to result from contextual response or healthy vaccine effect as there is no prevalent belief that vaccination improves AIRD outcomes, and SCCS utilises within-person comparisons accounting for the latter. However, this observation could result from regression to the mean.

We observed increased primary care consultation for joint pain and new corticosteroid prescriptions in the 14 days preceding vaccination. This could indicate opportunistic vaccine promotion to people consulting for AIRD flare. Alternatively, this may be due to the fact that most influenza vaccinations occur in late autumn and winter months ${ }^{5}$ that coincide with increased AIRD activity. It is therefore of interest that the 30 -day postvaccination period, also in the late autumn and winter months, expected to have more consultations for joint pain, had significantly fewer consultations.

The potential for vaccines to elicit an immune-mediated adverse reaction has raised concerns about a link between vaccines and AIRDs. ${ }^{9}{ }^{10}$ However, our data do not identify a significant association between vaccination and vasculitis and are in line with other studies. ${ }^{19}$ Similarly, there was no association between IIV and incident RA in the Epidemiological Investigation of Rheumatoid Arthritis cohort. ${ }^{20}$

The main strength of this study is its robust design, employing the SCCS method. By performing within-person comparisons, it minimises the influence of confounding between individuals, a serious problem in observational studies of adverse-effects following vaccination. Additionally, the use of consultation and prescription data minimised recall bias. The inclusion of a broad spectrum of AIRDs makes our findings generalisable. Additionally, we performed sensitivity analysis restricting to corticosteroid prescriptions on the same day as primary care consultation for either joint pain or RA flare.

However, there are several limitations to this study. First, data on disease activity is not recorded in the CPRD, and primary care consultations occur at least a few days after flare onset. Self-managed flares and those managed by rheumatologists are excluded. Thus, the use of consultation-based database underestimates the event rate. However, these caveats are unlikely to affect the validity of our findings as there is no reason for the ratio between events and primary care consultations to vary across the influenza-cycle. Our a priori decision to restrict the data analysis to one randomly selected influenza-cycle may have reduced the power for some outcomes such as new corticosteroid prescription which occurred in $\geq 2$ influenza cycles for $50 \%$ participants. This does not apply to other uncommon outcomes, about $90 \%$ of which occurred in only one influenza cycle.

In conclusion, this study supports the safety of influenza vaccine in AIRDs. These data should be used to address the fear of adverse effects from vaccination, an important reason for suboptimal uptake of influenza vaccination in AIRDs.

Contributors AA conceived the idea for the study and all authors planned the study collaboratively. AA, MG and GN developed the analysis plan; data analysis was undertaken by GN and supervised by MG and AA. JSN-V-T and PRM provided influenza specific input and advised on the data analysis plan. CDM provided primary care input. AA together with GN wrote the first draft of the manuscript. All authors reviewed the results and critically reviewed the manuscript for intellectual content. All authors approved the final version of the manuscript.

Funding This work was supported by Versus Arthritis (grant number 21297), formerly Arthritis Research UK, and the National Institute of Health Research (NIHRRP-2014-04-026)
Competing interests CDM is funded by the National Institute for Health Research (NIHR) Collaborations for Leadership in Applied Health Research and Care West Midlands, the NIHR School for Primary Care Research and an NIHR Research Professorship in General Practice (NIHR-RP-2014-04-026). JSN-V-T acknowledges funding from the NIHR Biomedical Research Centre (acute respiratory infections), $M D$ and $A A$ acknowledge funding from the NIHR Biomedical Research Centre (musculoskeletal theme) Nottingham. Potential conflicts of interest: JSN-V-T is currently on secondment to the Department of Health and Social Care, England (DHSC). The views expressed in this paper are those of the authors and do not necessarily represent those of the National Health Service, NIHR or DHSC. All other authors have declared no conflicts of interest.

Patient consent for publication Not required.

Ethics approval The study was approved by the Independent Scientific Advisory Committee (Reference: 16_288R).

Provenance and peer review Not commissioned; externally peer reviewed.

Data sharing statement Data may be obtained from a third party and are not publicly available.

Open access This is an open access article distributed in accordance with the Creative Commons Attribution 4.0 Unported (CC BY 4.0) license, which permits others to copy, redistribute, remix, transform and build upon this work for any purpose, provided the original work is properly cited, a link to the licence is given, and indication of whether changes were made. See: https://creativecommons.org/ licenses/by/4.0/.

\section{REFERENCES}

1 Blumentals WA, Arreglado A, Napalkov P, et al. Rheumatoid arthritis and the incidence of influenza and influenza-related complications: a retrospective cohort study. BMC Musculoskelet Disord 2012;13.

2 Chen C-M, Chen H-J, Chen W-S, et al. Clinical effectiveness of influenza vaccination in patients with rheumatoid arthritis. Int J Rheum Dis 2018;21:1246-53.

3 Nakafero G, Grainge MJ, Myles P, et al. Inactivated influenza vaccine prevents respiratory infections and improves all-cause and cause-specific mortality in immunosuppressed people with autoimmune rheumatic diseases: propensity score adjusted cohort study using data from clinical Practice Research Datalink. Arthritis \& Rheumatology 2018;70:1-3584.

4 Hua C, Barnetche T, Combe B, et al. Effect of methotrexate, anti-tumor necrosis factor $\alpha$, and rituximab on the immune response to influenza and pneumococcal vaccines in patients with rheumatoid arthritis: a systematic review and meta-analysis. Arthritis Care Res 2014;66:1016-26.

5 Nakafero G, Grainge MJ, Myles PR, et al. Predictors and temporal trend of flu vaccination in auto-immune rheumatic diseases in the UK: a nationwide prospective cohort study. Rheumatology 2018;57:1726-34.

6 Luque Ramos A, Hoffmann F, Callhoff J, et al. Influenza and pneumococcal vaccination in patients with rheumatoid arthritis in comparison with ageand sex-matched controls: results of a claims data analysis. Rheumatol Int 2016;36:1255-63

7 Loubet $\mathrm{P}$, Kernéis $\mathrm{S}$, Groh M, et al. Attitude, knowledge and factors associated with influenza and pneumococcal vaccine uptake in a large cohort of patients with secondary immune deficiency. Vaccine 2015;33:3703-8.

8 Bridges MJ, Coady D, Kelly CA, et al. Factors influencing uptake of influenza vaccination in patients with rheumatoid arthritis. Ann Rheum Dis 2003:62:685.

9 lyngkaran Pet al. Rheumatoid vasculitis following influenza vaccination. Rheumatology 2003;42:907-9.

10 Birck R, Kaelsch I, Schnuelle P, et al. ANCA-associated vasculitis following influenza vaccination: causal association or mere coincidence? J Clin Rheumatol 2009:15:289-91.

11 Kirchner E, Ruffing V. Barriers to immunizations and strategies to enhance immunization rates in adults with autoimmune inflammatory diseases. Rheumatic Disease Clinics of North America 2017:43:15-26.

12 Park JK, Lee YJ, Shin K, et al. Impact of temporary methotrexate discontinuation for 2 weeks on immunogenicity of seasonal influenza vaccination in patients with rheumatoid arthritis: a randomised clinical trial. Ann Rheum Dis 2018;77:898-904

13 Park JK, Lee MA, Lee EY, et al. Effect of methotrexate discontinuation on efficacy of seasonal influenza vaccination in patients with rheumatoid arthritis: a randomised clinical trial. Ann Rheum Dis 2017:76:1559-65.

14 Herrett E, Gallagher AM, Bhaskaran K, et al. Data resource profile: clinical Practice Research Datalink (CPRD). Int J Epidemiol 2015:44:827-36.

15 Whitaker HJ, Farrington CP, Spiessens B, et al. Tutorial in biostatistics: the selfcontrolled case series method. Stat Med 2006;25:1768-97.

16 Smeeth L, Thomas SL, Hall AJ, et al. Risk of myocardial infarction and stroke after acute infection or vaccination. N Eng/ J Med 2004;351:2611-8. 
17 Gross PA, Russo C, Dran S, et al. Time to earliest peak serum antibody response to influenza vaccine in the elderly. Clin Diagn Lab Immunol 1997;4:491-2.

18 Shrank WH, Patrick AR, Brookhart MA. Healthy user and related biases in observational studies of preventive interventions: a primer for physicians. J Gen Intern Med 2011;26:546-50
19 Watanabe T. Vasculitis following influenza vaccination: a review of the literature. Curr Rheumatol Rev 2017;13:188-96.

20 Bengtsson C, Kapetanovic MC, Källberg H, et al. Common vaccinations among adults do not increase the risk of developing rheumatoid arthritis: results from the Swedish EIRA study. Ann Rheum Dis 2010;69:1831-3. 